\title{
Singlet-doublet fermion and triplet scalar dark matter with radiative neutrino masses
}

\author{
Juri Fiaschi, ${ }^{a}$ Michael Klasen ${ }^{a}$ and Simon May ${ }^{a, b}$ \\ ${ }^{a}$ Institut für Theoretische Physik, Westfälische Wilhelms-Universität Münster, \\ Wilhelm-Klemm-Straße 9, 48149 Münster, Germany \\ ${ }^{b}$ Max-Planck-Institut für Astrophysik, \\ Karl-Schwarzschild-Straße 1, 85741 Garching, Germany \\ E-mail: fiaschi@uni-muenster.de, michael.klasen@uni-muenster.de, \\ simon.may@uni-muenster.de
}

ABSTRACT: We present a detailed study of a combined singlet-doublet fermion and triplet scalar model for dark matter. These models have only been studied separately in the past. Together, they form a simple extension of the Standard Model that can account for dark matter and explain the existence of neutrino masses, which are generated radiatively. This holds even if singlet-doublet fermions and triplet scalars never contribute simultaneously to the dark matter abundance. However, this also implies the existence of lepton flavour violating processes. In addition, this particular model allows for gauge coupling unification. The new fields are odd under a new $\mathbb{Z}_{2}$ symmetry to stabilise the dark matter candidate. We analyse the dark matter, neutrino mass and lepton flavour violation aspects both separately and in conjunction, exploring the viable parameter space of the model. This is done using a numerical random scan imposing successively the neutrino mass and mixing, relic density, Higgs mass, direct detection, collider and lepton flavour violation constraints. We find that dark matter in this model is fermionic for masses below about $1 \mathrm{TeV}$ and scalar above. The narrow mass regions found previously for the two separate models are enlarged by their coupling. While coannihilations of the weak isospin partners are sizeable, this is not the case for fermions and scalars despite their often similar masses due to the relatively small coupling of the two sectors, imposed by the small neutrino masses. We observe a high degree of complementarity between direct detection and lepton flavour violation experiments, which should soon allow to fully probe the fermionic dark matter sector and at least partially the scalar dark matter sector.

Keywords: Beyond Standard Model, Cosmology of Theories beyond the SM, Neutrino Physics, Discrete Symmetries

ArXiv EPrint: 1812.11133 


\section{Contents}

1 Introduction 1

2 Description of the model 3

3 Dark matter relic density $\quad 6$

3.1 Singlet-doublet fermions $\quad 6$

3.2 Triplet scalars 8

4 Radiative neutrino masses $\quad 9$

5 Dark matter direct detection and lepton flavour violation $\quad 12$

$\begin{array}{ll}5.1 \text { Singlet-doublet fermion dark matter } & 14\end{array}$

$\begin{array}{lll}5.2 & \text { Triplet scalar dark matter } & 15\end{array}$

$\begin{array}{llr}6 & \text { Conclusion } & 18\end{array}$

\section{Introduction}

Evidence for dark matter (DM) is accumulating from astrophysical observations at many different length scales [1]. Since no particle in the Standard Model (SM) of particle physics has the required properties of being uncharged under the electromagnetic and strong interactions and to be sufficiently cold to explain the observed large scale structure in the Universe, DM is widely believed to provide a strong indication for physics beyond the SM.

The simplest solution to the DM puzzle consists in adding a small number of new particles to the SM, out of which the lightest is neutral and stable, e. g. due to a discrete $\mathbb{Z}_{2}$ symmetry. Weakly interacting massive particles (WIMPs), such as heavy scalars or neutrinos, are among the most promising candidates, as they have the right cross section to be produced as thermal relics with the observed density $\Omega_{\mathrm{c}}^{\mathrm{obs}} h^{2}=0.120 \pm 0.001$ [2]. Here, $h$ denotes the present Hubble expansion rate in units of $100 \mathrm{~km} \mathrm{~s}^{-1} \mathrm{Mpc}^{-1}$. Many of these so-called minimal models have been studied in the past, including those with scalar singlets [3], doublets [4, 5], and triplets [6-10], fermion singlets [11, 12], doublets [13-15], and triplets [16], as well as with higher scalar [17] and/or fermion multiplets [18]. In particular, triplet scalar DM requires hypercharge $Y=0$ such that the $\mathrm{SU}(2)_{\mathrm{L}}$ component with $T_{3}=0$ is neutral [19]. On the other hand, mixing fermion singlets and doublets reduces the coupling to weak gauge bosons and can transform DM from a Dirac into a Majorana particle, yielding both the correct relic density and still allowed direct detection cross sections [13].

Particularly well motivated models are those that do not only provide a DM candidate, but also solve other SM problems such as the smallness of neutrino masses. This is possible 
when the $d=5$ Weinberg operator is realised at one loop [20], such that the particles in the loop have opposite $\mathbb{Z}_{2}$ parity to the SM particles and include a neutral DM candidate [19]. One of the most popular so-called radiative seesaw models is the scotogenic model with only one additional scalar (inert Higgs) $\mathrm{SU}(2)_{\mathrm{L}}$ doublet and a (right-handed neutrino) fermion singlet [21], for which we recently demonstrated the importance of coannihilations between the scalar dark matter and the right-handed neutrinos [22]. Many variants with general multiplets containing two scalars and one fermion have subsequently been proposed. In particular, the observed relic density was found to require a scalar triplet DM mass of $2.5 \mathrm{TeV}$ and couplings of $\mathcal{O}\left(10^{-3}\right)$ to an additional charged scalar singlet and vector-like doublet fermion to reproduce the observed neutrino mass differences [23, 24], whereas the scalar triplet DM mass could vary from $1.7 \mathrm{TeV}$ to $2.6 \mathrm{TeV}$ when a neutral scalar singlet and vector-like doublet fermion were added [23, 25]. Unfortunately, these masses/couplings are too large/small to explain the observed tension in the muon's $g-2$ between theory and experiment [26].

The models above all belong to the one-loop topology T3 with two scalars and one fermion $[19,20]$. In this paper, we study a model of topology T1-3 with one scalar and two fermions, one of which is vector-like. In contrast to the first of these models (T13-A with hypercharge parameter $\alpha=0$ ), where the scalar DM was a singlet, had to be lighter than $600 \mathrm{GeV}$, and where the parameter space will soon be explored by $\mu \rightarrow 3 e$ and $\mu-e$ conversion experiments [27], we investigate here a model (T1-3-B, also with $\alpha=0$ ) where the scalar DM is the neutral component of a triplet. In both cases, (at least) two generations of scalars must be introduced, so that not only the constraints from the DM relic density and observed Higgs mass, but also two non-zero neutrino mass differences and mixing angles can be imposed [28]. As for the model T1-3-A, we restrict ourselves to two scalar generations, which implies that the lightest neutrino is massless. Both models, like a previously studied model with both singlet-doublet scalars and fermions (T1-2-A with $\alpha=0$ ) [29], have the additional advantage that they allow for gauge coupling unification at a scale $\Lambda=\mathcal{O}\left(10^{13} \mathrm{GeV}\right)$, at variance with most of the other models mentioned above including the original scotogenic model [30]. Like all models classified in [19], these models feature Majorana neutrinos.

From the list of models with DM, radiative neutrino masses and gauge coupling unification which have not been studied previously, our model features the smallest number (six) of new interaction terms, which makes it possible to fully explore its parameter space. We do so by constructing the most general Lagrangian from the field content, eliminating a redundant term, generating model files for SPheno and micrOMEGAs with SARAH 4.13.0 [31], imposing the neutrino masses using the Casas-Ibarra parametrisation [32], and calculating the physical particle spectrum and relevant precision observables with SPheno 4.0.3 [33] as well as the DM relic density and direct detection cross sections with micrOMEGAs 4.3.5 [34]. The constructed Lagrangian was checked and template SARAH model files were generated by the newly-developed tool minimal-lagrangians, which is available upon request.

The remainder of this paper is organised as follows: in section 2 we introduce the model under examination, giving details of its field content and the full Lagrangian, describing the mixing of fundamental particles into physical states in the fermion and scalar sectors 


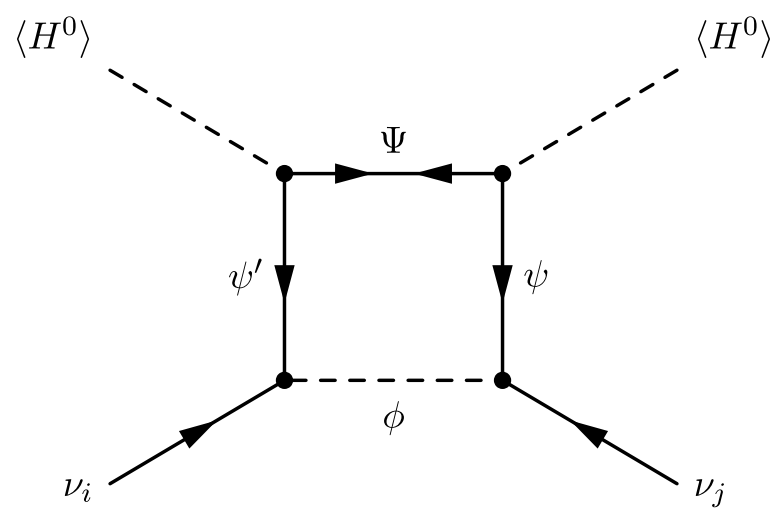

Figure 1. Loop topology T1-3 for the radiative generation of neutrino masses at the one-loop level.

\begin{tabular}{|cccccccc|}
\hline Field & Generations & Spin & Lorentz rep. & $\mathrm{SU}(3)_{\mathrm{C}}$ & $\mathrm{SU}(2)_{\mathrm{L}}$ & $\mathrm{U}(1)_{\mathrm{Y}}$ & $\mathbb{Z}_{2}$ \\
\hline$\Psi$ & 1 & $1 / 2$ & $(1 / 2,0)$ & $\mathbf{1}$ & $\mathbf{1}$ & 0 & -1 \\
$\psi$ & 1 & $1 / 2$ & $(1 / 2,0)$ & $\mathbf{1}$ & $\mathbf{2}$ & -1 & -1 \\
$\psi^{\prime}$ & 1 & $1 / 2$ & $(1 / 2,0)$ & $\mathbf{1}$ & $\mathbf{2}$ & 1 & -1 \\
$\phi_{i}$ & 2 & 0 & $(0,0)$ & $\mathbf{1}$ & $\mathbf{3}$ & 0 & -1 \\
\hline
\end{tabular}

Table 1. New fields and their quantum numbers in the model T1-3-B with $\alpha=0$.

as well as the mass splitting between neutral and charged states. In section 3, we discuss the features of the two distinct DM candidates within our model and update and clarify contradictory results in the literature. In section 4 we connect the dark sector to the radiative generation of neutrino masses and explain how we impose neutrino constraints on the parameter space of the model. In section 5 we study their impact on the nature, parameter space and direct detection prospects of the DM candidates and make predictions for the automatically generated lepton flavour violating (LFV) processes. Our conclusions are given in section 6 .

\section{Description of the model}

Following the notation in refs. [19, 20], the model T1-3-B with hypercharge parameter $\alpha=0$ is defined by extending the SM with the colour-singlet fields in table 1. T1-3 labels the general one-loop topology generating radiative neutrino masses, ${ }^{1}$ as shown in figure 1 , while T1-3-B is a specific $\mathrm{SU}(2)_{\mathrm{L}}$ multiplet structure of the new fields. The parameter $\alpha$ is simply a common offset to the hypercharge values given in table 1 - for example, in the model T1-3-B with $\alpha=2$, every value in the column $\mathrm{U}(1)_{\mathrm{Y}}$ is increased by 2 compared to T1-3-B with $\alpha=0$.

The present model therefore combines the $\mathrm{SU}(2)_{\mathrm{L}}$ triplet scalars $\phi_{i}$ of zero hypercharge $\left(\mathrm{U}(1)_{\mathrm{Y}}\right)$ with an $\mathrm{SU}(2)_{\mathrm{L}}$ singlet fermion $\Psi$ and doublet fermions $\psi, \psi^{\prime}$. They are all odd under the discrete symmetry $\mathbb{Z}_{2}$, while the SM fields are even. The components of the new

\footnotetext{
${ }^{1}$ The three topologies T1-1, T1-2 and T1-3, collectively T1- $n$, differ only in the number $n$ of fermion lines (as opposed to scalar lines) in the loop.
} 
fields are given by

$$
\Psi=\Psi^{0}, \quad \psi=\left(\begin{array}{c}
\psi^{0} \\
\psi^{-}
\end{array}\right), \quad \psi^{\prime}=\left(\begin{array}{c}
\psi^{+} \\
\psi^{\prime 0}
\end{array}\right), \quad \phi_{i}=\left(\begin{array}{cc}
\frac{1}{\sqrt{2}} \phi_{i}^{0} & \phi_{i}^{+} \\
\phi_{i}^{-} & -\frac{1}{\sqrt{2}} \phi_{i}^{0}
\end{array}\right)
$$

where superscripts indicate electric charges. To obtain two non-zero neutrino mass differences, two generations of scalar triplets are required. Since the scalar triplets have zero hypercharge, they are treated as real, $\left(\phi_{i}^{0}\right)^{\dagger}=\phi_{i}^{0},\left(\phi_{i}^{+}\right)^{\dagger}=\phi_{i}^{-} . \Psi$ has the same quantum numbers as a $\mathbb{Z}_{2}$-odd right-handed neutrino, whereas $\psi$ and $\psi^{\prime}$ together form a $\mathbb{Z}_{2}$-odd vector-like lepton doublet, which makes the model automatically anomaly-free. In principle, all neutral field components are possible DM candidates. The $\mathbb{Z}_{2}$ symmetry not only stabilises the lightest new particle against decay into SM fields, but also forbids neutrino masses from a tree-level type-I seesaw mechanism.

The most general renormalisable Lagrangian for the model is ${ }^{2}$

$$
\begin{aligned}
\mathcal{L}= & \mathcal{L}_{\mathrm{SM}}+\mathcal{L}_{\text {kin }}-\frac{1}{2}\left(M_{\phi}^{2}\right)^{i j} \operatorname{Tr}\left(\phi_{i} \phi_{j}\right)-\left(\frac{1}{2} M_{\Psi} \Psi \Psi+\text { H. c. }\right)-\left(M_{\psi \psi^{\prime}} \psi \psi^{\prime}+\text { H. c. }\right) \\
& -\left(\lambda_{2}\right)^{i j} H^{\dagger} \phi_{i} \phi_{j} H-\left(\lambda_{3}\right)^{i j k m} \operatorname{Tr}\left(\phi_{i} \phi_{j} \phi_{k} \phi_{m}\right) \\
& -\left(\lambda_{4}\left(H^{\dagger} \psi^{\prime}\right) \Psi+\text { H. c. }\right)-\left(\lambda_{5}(H \psi) \Psi+\text { H. c. }\right)-\left(\left(\lambda_{6}\right)^{i j} L_{i} \phi_{j} \psi^{\prime}+\text { H. c. }\right),
\end{aligned}
$$

where $H$ is the SM Higgs field (with vacuum expectation value $v$ and quartic coupling $\lambda)^{3}$ and we have combined the term

$$
-\left(\lambda_{1}\right)^{i j}\left(H^{\dagger} H\right) \operatorname{Tr}\left(\phi_{i} \phi_{j}\right)
$$

with the one proportional to $\lambda_{2}$ using the identity

$$
H^{\dagger}\left\{\phi_{i}, \phi_{j}\right\} H=\left(H^{\dagger} H\right) \operatorname{Tr}\left(\phi_{i} \phi_{j}\right)
$$

However, for the remaining analysis, we will instead use the $\lambda_{1}$ term (2.3) for easier comparison with the previous literature on models with one scalar triplet, which is completely equivalent as long as there are no CP-violating phases. The couplings $\lambda_{4}$ and $\lambda_{5}$ have the function of Yukawa terms, which link the fermion singlet and doublets to the SM Higgs boson. The coupling $\lambda_{6}$ connects the SM lepton doublet $L$ to the new fields, so that it will be involved in the process of radiative neutrino masses generation. ${ }^{4}$

In order to ensure that the theory has a stable vacuum, the scalar potential in (2.2) should have a minimum, i.e. it should be bounded from below. For the SM Higgs field with a single (inert) scalar triplet (one generation), constraints on the parameters following from

\footnotetext{
${ }^{2}$ Here and in the following, products of field multiplets are always defined to be Lorentz- and gaugeinvariant. For example, the product of the two $\mathrm{SU}(2)_{\mathrm{L}}$ Weyl spinors $\psi$ and $\psi^{\prime}$ should be interpreted as $\psi \psi^{\prime}=\varepsilon^{j i} \varepsilon^{j^{\prime} i^{\prime}} \psi_{i i^{\prime}} \psi_{j j^{\prime}}^{\prime}$, where the indices represent the $\mathrm{SU}(2)_{\mathrm{L}}$ and spinor components.

${ }^{3}$ We have used a value of $\lambda=0.2612$ as an input to SPheno throughout the analysis.

${ }^{4}$ Except for $\left(M_{\phi}^{2}\right)^{i j}, \lambda_{1}^{i j}, \lambda_{3}^{i j} \in \mathbb{R}$, the parameters can, in principle, have a complex CP-violating phase. Note that not all of the parameters are independent: $\left(M_{\phi}^{2}\right)^{i j}=\left(M_{\phi}^{2}\right)^{j i},\left(\lambda_{1}\right)^{i j}=\left(\lambda_{1}\right)^{j i},\left(\lambda_{2}\right)^{i j *}=\left(\lambda_{2}\right)^{j i}$, $\left(\lambda_{3}\right)^{i j k m}=\left(\lambda_{3}\right)^{j k m i}=\left(\lambda_{3}\right)^{k m i j}=\left(\lambda_{3}\right)^{m i j k}$.
} 
this requirement are given in $[6,17]$. In general, determining whether a function is bounded from below is not a trivial task and expressing the constraints on the parameters as analytic conditions is usually only possible for specific directions in the variables. Constraints analogous to those for one generation can be obtained:

$$
\lambda_{3}^{i i i i} \geq 0, \quad 2 \sqrt{\lambda \lambda_{3}^{i i i i}} \geq-\lambda_{1}^{i i}
$$

In the direction where all fields are taken to infinity equally with only some possible signs between them, the condition

$$
\lambda+\lambda_{1}^{11}+\lambda_{1}^{22}+\lambda_{3}^{1111}+\lambda_{3}^{2222}+\lambda_{3}^{1212}+2 \lambda_{3}^{1112} \geq 2\left|\lambda_{1}^{12}\right|+4\left(\left|\lambda_{3}^{1112}\right|+\left|\lambda_{3}^{1222}\right|\right)
$$

must hold. For $H=0$, the potential has the same form as that in [35] (with $d=0$ ), so additional conditions can be obtained analogously, such as:

$$
\begin{aligned}
\sqrt{\lambda_{3}^{1111} \lambda_{3}^{2222}} & \geq \lambda_{3}^{1212}-2 \lambda_{3}^{1122}, \\
\lambda_{3}^{1111}+\lambda_{3}^{2222}+\lambda_{3}^{1212}+2 \lambda_{3}^{1122} & \geq 4\left(\left|\lambda_{3}^{1112}\right|+\left|\lambda_{3}^{1222}\right|\right), \\
\lambda_{3}^{1111} \lambda_{3}^{2222}+\frac{1}{2} \sqrt{\lambda_{3}^{1111} \lambda_{3}^{2222}}\left(\lambda_{3}^{1212}+2 \lambda_{3}^{1122}\right) & \geq 2\left(\lambda_{3}^{1111}\left|\lambda_{3}^{1112}\right|+\lambda_{3}^{2222}\left|\lambda_{3}^{1222}\right|\right) .
\end{aligned}
$$

To summarize, vacuum stability can be controlled through the parameter $\lambda_{3}$, which does however not affect the DM phenomenology (see below). Apart from loop corrections, the vacuum structure is otherwise unchanged since the imposed exact $\mathbb{Z}_{2}$ symmetry prevents the new scalars $\phi_{i}$ from acquiring nonzero vacuum expectation values.

After electroweak symmetry breaking, the mass terms of the neutral fermions take the form

$$
\mathcal{L}_{\mathrm{f}, 0}=-\frac{1}{2} M_{\Psi} \Psi \Psi-M_{\psi \psi^{\prime}} \psi^{0} \psi^{\prime 0}-\frac{\lambda_{4} v}{\sqrt{2}} \psi^{\prime 0} \Psi-\frac{\lambda_{5} v}{\sqrt{2}} \psi^{0} \Psi+\text { H. c. }
$$

where $v$ is the vacuum expectation value of the SM Higgs field $H$. Diagonalisation of the mass matrix $[13,14]$

$$
M_{\mathrm{f}, 0}=\left(\begin{array}{ccc}
M_{\Psi} & \frac{\lambda_{5} v}{\sqrt{2}} & \frac{\lambda_{4} v}{\sqrt{2}} \\
\frac{\lambda_{5} v}{\sqrt{2}} & 0 & M_{\psi \psi^{\prime}} \\
\frac{\lambda_{4} v}{\sqrt{2}} & M_{\psi \psi^{\prime}} & 0
\end{array}\right)
$$

with the unitary mixing matrix $U_{\chi}$ allows then to rewrite the neutral mass Lagrangian

$$
\mathcal{L}_{\mathrm{f}, 0}=-\frac{1}{2}\left(\Psi, \psi^{0}, \psi^{\prime 0}\right) M_{\mathrm{f}, 0}\left(\begin{array}{c}
\Psi \\
\psi^{0} \\
\psi^{\prime 0}
\end{array}\right)+\text { H.c. }=-\frac{1}{2}\left(\chi^{0}\right)^{\top} U_{\chi}^{*} M_{0} U_{\chi}^{\dagger} \chi^{0}+\text { H.c. }
$$

with

$$
U_{\chi}^{*} M_{\mathrm{f}, 0} U_{\chi}^{\dagger}=\operatorname{diag}\left(m_{\chi_{1}^{0}}, m_{\chi_{2}^{0}}, m_{\chi_{3}^{0}}\right)
$$

in terms of the physical Majorana fermions

$$
\chi^{0}=U_{\chi}\left(\begin{array}{c}
\Psi^{0} \\
\psi^{0} \\
\psi^{\prime 0}
\end{array}\right)
$$


For the two generations of scalar triplets, electroweak symmetry breaking induces a shift in the mass matrices

$$
M_{\phi^{0}}^{2}=M_{\phi^{ \pm}}^{2}=M_{\phi}^{2}+\lambda_{1} v^{2} .
$$

Note that at tree level, the charged and neutral mass matrices are identical. Similarly to the neutral fermions, the scalar mass eigenstates

$$
\eta^{0, \pm}=O_{\eta}\left(\begin{array}{c}
\phi_{1}^{0, \pm} \\
\phi_{2}^{0, \pm}
\end{array}\right)
$$

are obtained by diagonalising the scalar mass matrices with (in this case orthogonal) rotation matrices $O_{\eta}$, leading to

$$
O_{\eta} M_{\phi^{0, \pm}}^{2} O_{\eta}^{\top}=\operatorname{diag}\left(m_{\eta_{1}^{0, \pm}}^{2}, m_{\eta_{2}^{0, \pm}}^{2}\right) .
$$

At the one-loop level, gauge interactions introduce mass splittings

$$
\Delta m_{\eta_{i}}=m_{\eta_{i}^{ \pm}}-m_{\eta_{i}^{0}}=166 \mathrm{MeV}
$$

which, together with the new interactions, render the neutral components slightly lighter than the corresponding charged ones [18], making them potential dark matter candidates.

\section{Dark matter relic density}

Depending on the spin of the lightest neutral $\mathbb{Z}_{2}$-odd mass eigenstate, our model allows for both singlet-doublet fermion and triplet scalar DM. As mentioned in the introduction, these models have been studied separately in the past, which, however, prohibits the radiative generation of neutrino masses. In this section, we briefly comment on both scenarios individually, updating and clarifying the results in the literature. We will limit ourselves to one generation of scalar triplets in this section, which can be viewed as decoupling the second generation via

$$
\left(M_{\phi}^{2}\right)^{22}=(1000 \mathrm{TeV})^{2}, \quad\left(M_{\phi}^{2}\right)^{12}=\left(\lambda_{1}\right)^{i 2}=\left(\lambda_{6}\right)^{i 2}=0,
$$

since the scalars are decoupled in section 3.1 anyway and the additional generation does not introduce any qualitatively new phenomena in section 3.2 .

\subsection{Singlet-doublet fermions}

We first assume that DM is composed of the lightest neutral fermion $\left(\chi_{1}^{0}\right)$. The scalar sector is decoupled by setting

$$
\left(M_{\phi}^{2}\right)^{11}=(1000 \mathrm{TeV})^{2}, \quad \lambda_{1}=\lambda_{3}=\lambda_{6}=0 .
$$

Choosing $M_{\Psi}=200 \mathrm{GeV}, M_{\psi \psi^{\prime}}=300 \mathrm{GeV}$ and $\lambda_{5}=0.36$ and neglecting loop corrections, we generally reproduce the relic density and direct detection cross sections in figure 2 of ref. [13]. In particular, depending on the value of $\lambda_{4}$ and thus the singlet-doublet fermion 


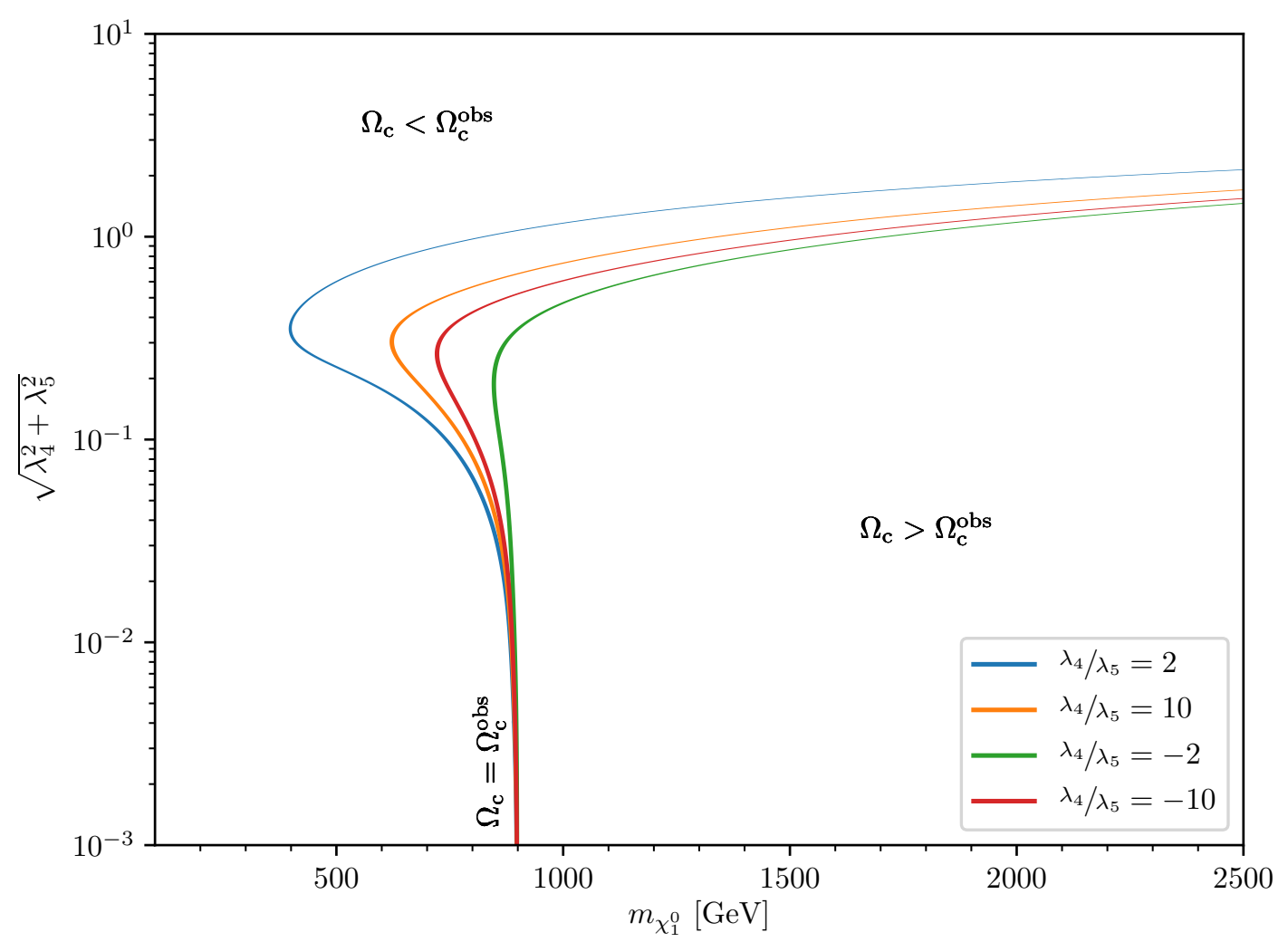

Figure 2. The observed relic density contours $\left(\Omega_{\mathrm{c}}^{\mathrm{obs}} h^{2}\right)$, separating regions of too small and too large relic density, as a function of the singlet-doublet fermion DM mass $m_{\chi_{1}^{0}}$ and the Yukawa couplings $\sqrt{\lambda_{4}^{2}+\lambda_{5}^{2}}$ assuming $M_{\Psi}=M_{\psi \psi^{\prime}}$ for different values of $\lambda_{4} / \lambda_{5}$.

mixing, blind spots of spin-independent and -dependent direct detection experiments with no DM couplings to Higgs and $Z$ bosons appear. Note, however, that our relic density exhibits a slightly higher peak value when the (now known) Higgs mass of $125.08 \mathrm{GeV}$ [28] is used. Also, our spin-independent direct detection cross section is smaller by about a factor of two, which we can trace to updated nuclear form factors in micrOMEGAs (version 4.3.5 vs. 2.4). A similar observation also holds for the fermion mass scans in figure 5 of ref. [14], where $\lambda_{4} / \lambda_{5} \in\{ \pm 2, \pm 10\}$ and $\sqrt{\lambda_{4}^{2}+\lambda_{5}^{2}}=0.3$. While we reproduce the relic density and spin-dependent detection contours, our spin-independent detection contours are shifted by about $0.1 \mathrm{TeV}$ even after adjusting for the nuclear form factors. Furthermore, the latest XENON1T results [36] almost double the size of the excluded low-mass regions, so that, e. g., $M_{\Psi} \simeq M_{\psi \psi^{\prime}}>1 \mathrm{TeV}$ for $\lambda_{4} / \lambda_{5}=2$. Below masses of $1 \mathrm{TeV}$, the observed relic density requires $M_{\Psi} \simeq M_{\psi \psi^{\prime}}$ independently of $\lambda_{4} / \lambda_{5}$. For higher singlet masses, DM becomes a pure doublet with constant mass $1 \mathrm{TeV}$. For other, in particular negative values of $\lambda_{4} / \lambda_{5}$, even the new XENON1T limits become as weak as $200 \mathrm{GeV}$.

Figure 2 shows another example illustrating the behavior of the singlet-doublet fermion model for the special case $M_{\Psi}=M_{\psi \psi^{\prime}}$. For small Yukawa couplings, both mixing between the neutral fermions and cross sections for annihilation into gauge bosons (through the mixing angles) and the Higgs boson are small. On the other hand, for $\sqrt{\lambda_{4}^{2}+\lambda_{5}^{2}} \geq 0.4$, the 


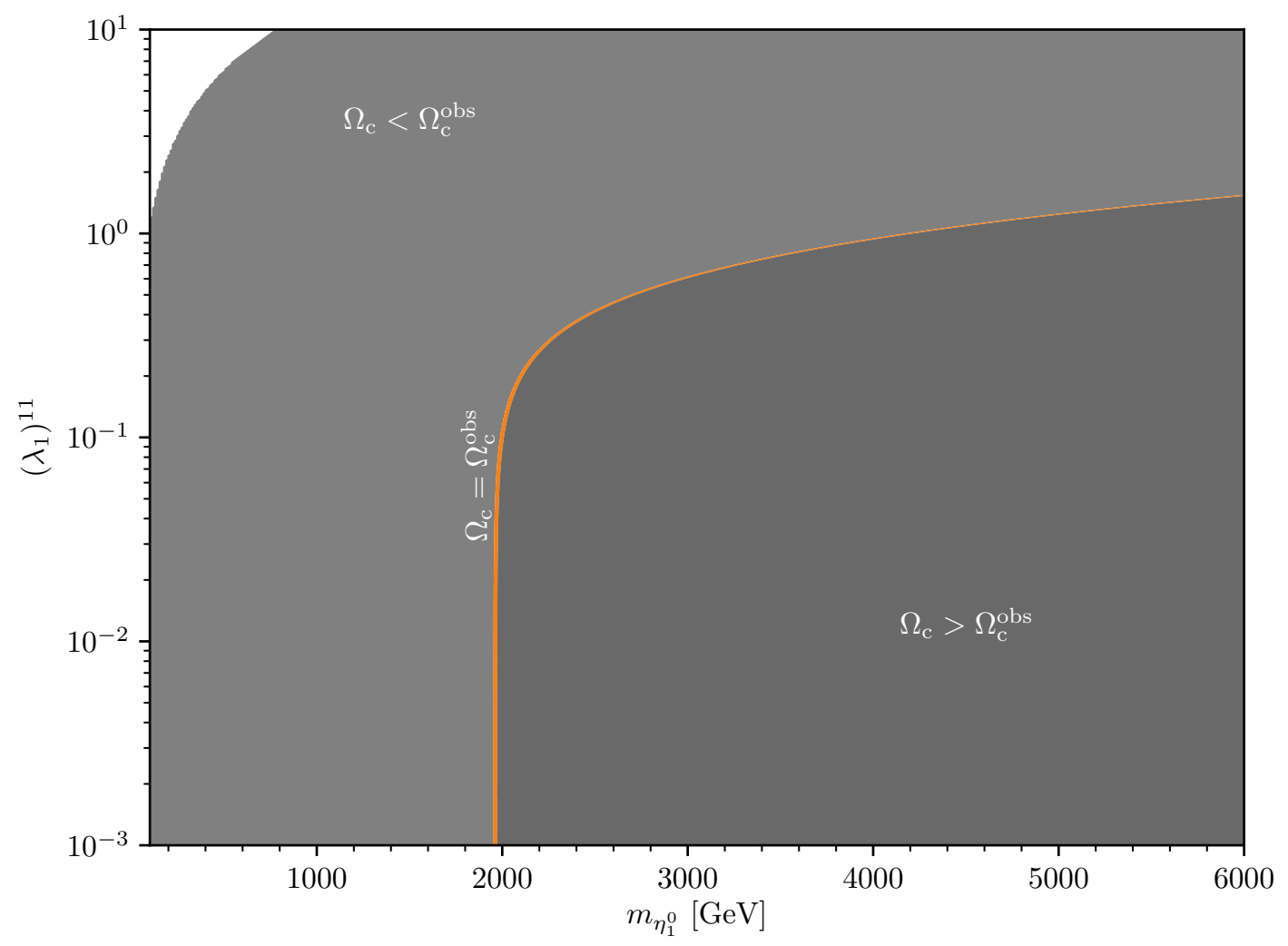

Figure 3. The observed relic density contour $\left(\Omega_{\mathrm{c}}^{\mathrm{obs}} h^{2}\right)$, separating regions of too small (above) and too large (below) relic density, as a function of the triplet scalar DM mass $m_{\eta_{1}^{0}}$ and its Higgs coupling $\left(\lambda_{1}\right)^{11}$. Here, we assume only one generation of scalars.

DM mass must rise quickly to compensate the increasingly efficient annihilation channels. The mass splitting depends on the relative size of the couplings $\lambda_{4} / \lambda_{5}$, controlling the impact of coannihilation processes.

\subsection{Triplet scalars}

The literature in the case when DM is composed of the lightest neutral scalar $\left(\eta_{1}^{0}\right)$ is unfortunately contradictory, and we take the opportunity to clarify the situation. We therefore now decouple the fermion sector by setting

$$
M_{\Psi}=M_{\psi \psi^{\prime}}=1000 \mathrm{TeV}, \quad \lambda_{4}=\lambda_{5}=\lambda_{6}=0 .
$$

Furthermore, we find that the parameter $\lambda_{3}$ inducing scalar co-annihilations and conversions does not play an important role, so that we not only neglect loop corrections, but also set $\lambda_{3}=0$ in this section.

For one generation, the so-called inert triplet model was first studied in ref. [18] (although only including gauge interactions) with the result that DM had to be in a very narrow mass region of $(2.00 \pm 0.05) \mathrm{TeV}$ (see also refs. $[7,8,10,17])$ and that the spinindependent cross section was $1.3 \times 10^{-9} \mathrm{pb}$. For large couplings $\lambda_{1} \simeq 1$ of $\mathrm{DM}$ to Higgs bosons, a DM mass increase by about $20 \%$ was predicted due the opening of Higgs channels 


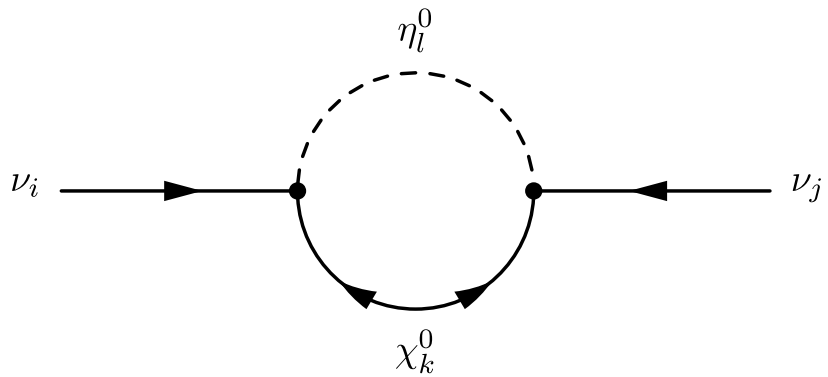

Figure 4. Feynman diagram for the radiative generation of neutrino masses at the one-loop level after electroweak symmetry breaking and mixing $\left(k \in\left\{1, \ldots, n_{\mathrm{f}}\right\}, l \in\left\{1, \ldots, n_{\mathrm{s}}\right\}\right)$. The convention for Feynman diagrams is the same as in ref. [37].

in addition to the dominant DM annihilation into weak gauge bosons. Figure 3 confirms that $m_{\eta_{1}^{0}} \simeq 2 \mathrm{TeV}$ for $\lambda_{1} \leq 0.2$, but also shows that the Higgs channels then become so efficient that the DM mass rapidly increases into the multi-TeV region (cf. figure 5 of ref. [17] and figure 4 of ref. [8]). Qualitatively, this behaviour was also found in figure 1 of ref. [6], but with a significant shift to a larger DM mass of $5.5 \mathrm{TeV}$. Correcting the normalisation of the squared neutral mass in their equation (7) by a factor of two can partially explain this discrepancy. With the same (incorrect) normalisation, an even larger DM mass of $7 \mathrm{TeV}$ was found, but not commented upon in ref. [9]. Surprisingly, and despite using more recent nuclear form factors, we can reproduce the spin-independent detection cross sections in figure 2 of ref. [6], which also agree roughly with the numbers given in refs. [17, 18] for sufficiently small $\lambda_{1} \simeq 0.01$. As described there, gauge interactions increase the cross section at one loop by $0.86 \times 10^{-9} \mathrm{pb}$, while the Sommerfeld effect induces a shift towards slightly larger DM masses (cf. figure 6 of ref. [17]).

\section{Radiative neutrino masses}

After electroweak symmetry breaking, neutrino masses in our model arise from the oneloop diagram shown in figure 4 . Only the $n_{\mathrm{s}}$ neutral scalar and $n_{\mathrm{f}}$ neutral fermion fields contribute to mass generation, whereas the charged fields enter only into the propagator correction. In our model, the $n_{\mathrm{f}}=3$ fermions are superpositions of $\mathrm{SU}(2)_{\mathrm{L}}$ singlets and doublets, while the $n_{\mathrm{s}}=2$ scalars are superpositions of the two generations of scalar triplets required for two non-zero neutrino masses. Evaluating the two-point function in dimensional regularisation and summing over all contributions leads to

$$
\begin{aligned}
\left(M_{\nu}\right)_{i j} & =\frac{1}{32 \pi^{2}} \sum_{l=1}^{n_{\mathrm{s}}} \lambda_{6}^{i m} \lambda_{6}^{j n}\left(O_{\eta}\right)_{l m}\left(O_{\eta}\right)_{l n} \sum_{k=1}^{n_{\mathrm{f}}}\left(U_{\chi}\right)_{k 3}^{*} \frac{m_{\chi_{k}^{0}}^{3}}{m_{\eta_{l}^{0}}^{2}-m_{\chi_{k}^{0}}^{2}} \ln \left(\frac{m_{\chi_{k}^{0}}^{2}}{m_{\eta_{l}^{0}}^{2}}\right) \\
& =\frac{1}{32 \pi^{2}} \sum_{l=1}^{n_{\mathrm{s}}} A_{l} \lambda_{6}^{i m} \lambda_{6}^{j n}\left(O_{\eta}\right)_{l m}\left(O_{\eta}\right)_{l n}
\end{aligned}
$$

where the ultraviolet divergences have cancelled as expected for a renormalisable model with vanishing neutrino masses at tree level. As evident from eq. (4.1), the structure of 


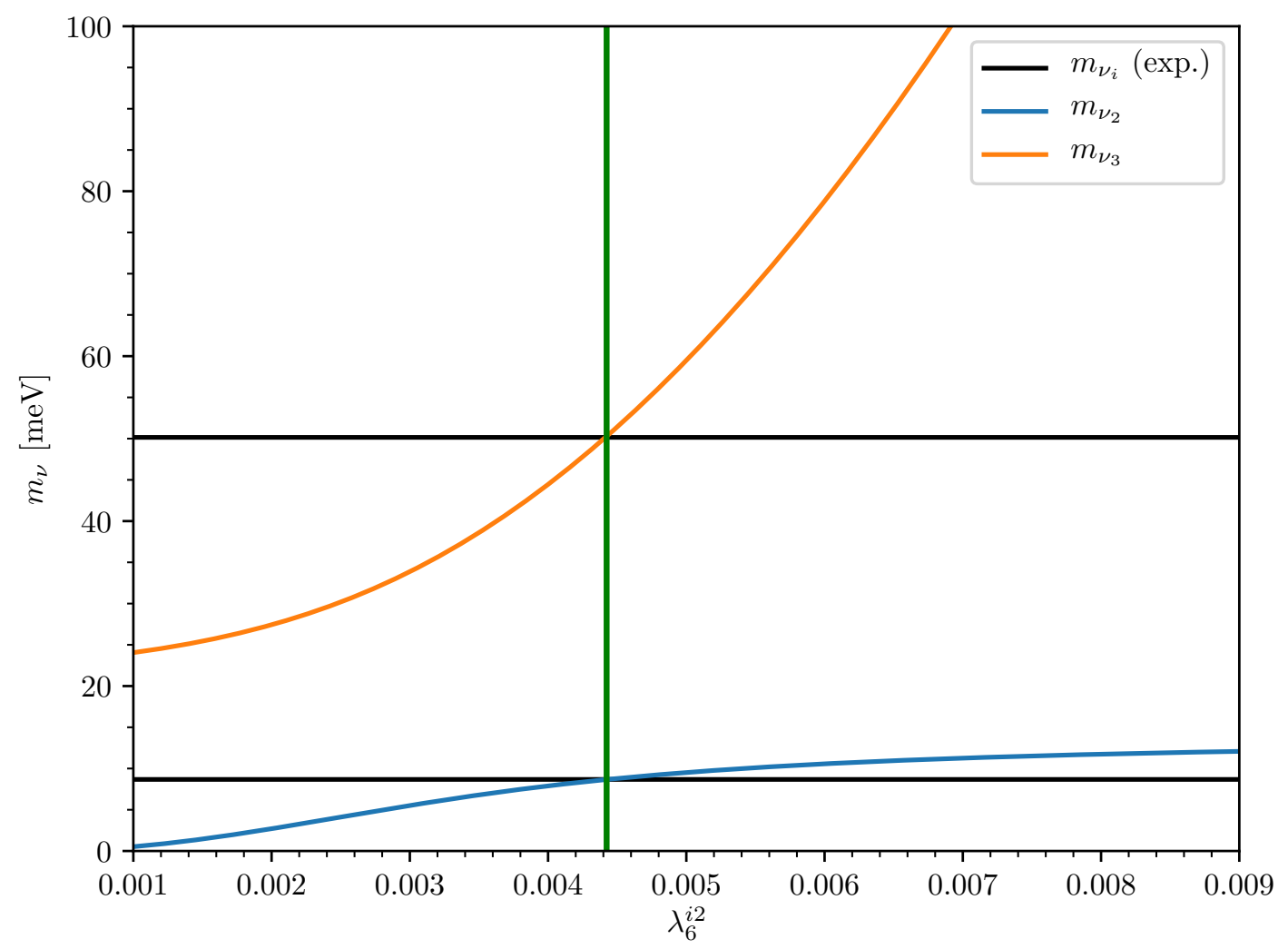

Figure 5. Neutrino masses $m_{\nu_{i}}$ in the model T1-3-B $(\alpha=0)$ with two generations of scalar triplets as a function of $\lambda_{6}^{i 2}$. The used parameters are given in table 2. The experimentally determined neutrino masses are reproduced for $\lambda_{6}^{i 2}=0.0044$.

the mass matrix is chiefly determined by the number of scalar generations, which must be at least as large as the desired number of non-zero neutrino masses.

The symmetric neutrino mass matrix $M_{\nu}$ in eq. (4.1) is diagonalised by the PontecorvoMaki-Nakagawa-Sakata (PMNS) matrix $U_{\nu}$

$$
U_{\nu}^{\dagger} M_{\nu} U_{\nu}=D_{\nu}=\operatorname{diag}\left( \pm m_{\nu_{1}}, \pm m_{\nu_{2}}, \pm m_{\nu_{3}}\right)
$$

Since the neutrino mass matrix is of the same form as in model T1-3-A with hypercharge parameter $\alpha=0$ [27], it can similarly be expanded in the limit of small Yukawa couplings $\lambda_{4}, \lambda_{5} \ll 1$. At leading order, we obtain

$$
M_{\nu} \approx 100 \mathrm{meV} \frac{M_{\Psi}}{1 \mathrm{TeV}}\left(\frac{\lambda_{6}^{i j} \lambda_{4,5}}{10^{-5}}\right)^{2},
$$

i.e. for meV-scale neutrinos only the product $\lambda_{6}^{i j} \lambda_{4,5}$ has to be of order $10^{-5}$. As an example, we show in figure 5 the dependence of the two non-zero neutrino masses $m_{\nu_{i}}(i \in\{2,3\})$ on the scalar-fermion coupling $\lambda_{6}^{i 2}$. The mass parameters and other couplings used in this figure are listed in table 2 . 


\begin{tabular}{|lr|}
\hline Parameter & Value \\
\hline$\left(M_{\phi}\right)^{11}$ & $1.4 \mathrm{TeV}$ \\
$\left(M_{\phi}\right)^{22}$ & $3 \mathrm{TeV}$ \\
$\left(M_{\phi}\right)^{12}$ & 0 \\
$M_{\Psi}$ & $1 \mathrm{TeV}$ \\
$M_{\psi \psi^{\prime}}$ & $1.5 \mathrm{TeV}$ \\
$\lambda_{1}, \lambda_{3}$ & 0 \\
$\lambda_{4}, \lambda_{5}$ & $5 \times 10^{-3}$ \\
$\lambda_{6}^{i 1}(i \neq 3)$ & $3 \times 10^{-4}$ \\
$\lambda_{6}^{3 l}$ & $3.05 \times 10^{-3}$ \\
\hline
\end{tabular}

Table 2. Mass parameters and couplings used in figure 5.

The neutrino mass matrix $M_{\nu}$ depends explicitly on the couplings

$$
\lambda_{6}=\left(\begin{array}{ll}
\lambda_{6}^{e 1} & \lambda_{6}^{e 2} \\
\lambda_{6}^{\mu 1} & \lambda_{6}^{\mu 2} \\
\lambda_{6}^{\tau 1} & \lambda_{6}^{\tau 2}
\end{array}\right)
$$

and on the masses $m_{\eta_{l}^{0}}$ and $m_{\chi_{k}^{0}}$ of the neutral scalars and fermions, while the dependence on the other couplings $\lambda_{1,4,5}$ remains implicit in the mixing matrices. The Casas-Ibarra parametrisation [32] then allows to obtain $\lambda_{6}$ from the experimental neutrino data, once the other couplings and masses have been fixed. The procedure is as follows: first, one factorises the explicit dependence on the couplings $\lambda_{6}$ from the neutrino mass matrix in eq. (4.1),

$$
M_{\nu}=\lambda_{6} M \lambda_{6}^{\top}=\lambda_{6} U_{M}^{\dagger} D_{M} U_{M} \lambda_{6}^{\top},
$$

where in the last step the $n_{\mathrm{s}} \times n_{\mathrm{s}}$ matrix $M$ has been diagonalised to the matrix $D_{M}$ using the unitary (here: orthogonal, as we have neglected complex phases) matrix $U_{M}$. On the other hand, the neutrino mass matrix can be diagonalised using the PMNS matrix as in eq. (4.2). Combining these expressions, one obtains

$$
D_{\nu}=U_{\nu}^{\dagger} \lambda_{6} U_{M}^{\dagger} D_{M} U_{M} \lambda_{6}^{\top} U_{\nu}
$$

or, equivalently $\left(m_{\nu_{1}}=0\right),{ }^{5}$

$$
\operatorname{diag}(0,1,1)=D_{\nu}^{-\frac{1}{2}} U_{\nu}^{\dagger} \lambda_{6} U_{M}^{\dagger} D_{M} U_{M} \lambda_{6}^{\top} U_{\nu} D_{\nu}^{-\frac{1}{2}}
$$

The left-hand side can be rewritten as $\operatorname{diag}(0,1,1)=R^{\dagger} R$ with the "rotation matrix"

$$
R=\left(\begin{array}{ccc}
0 & \cos (\theta) & \sin (\theta) \\
0 & -\sin (\theta) & \cos (\theta)
\end{array}\right),
$$

\footnotetext{
${ }^{5}$ For a diagonal matrix $D, D^{ \pm \frac{1}{2}}=\operatorname{diag}\left(D_{11}^{ \pm \frac{1}{2}}, D_{22}^{ \pm \frac{1}{2}}, \ldots\right)$.
} 


\begin{tabular}{|lrr|}
\hline Parameter & Fermion DM & Scalar DM \\
\hline$\left(M_{\phi}\right)^{11}$ & $3 \mathrm{TeV}$ & {$[0.5 \mathrm{TeV}, 3.5 \mathrm{TeV}]$} \\
$\left(M_{\phi}\right)^{22}$ & $1000 \mathrm{TeV}$ & $1000 \mathrm{TeV}$ \\
$\left(M_{\phi}\right)^{12}$ & 0 & 0 \\
$M_{\Psi}$ & {$[0.5 \mathrm{TeV}, 2.8 \mathrm{TeV}]$} & $3 \mathrm{TeV}$ \\
$M_{\psi \psi^{\prime}}$ & $M_{\Psi}$ & $2.8 \mathrm{TeV}$ \\
$\lambda_{1}, \lambda_{3}, \lambda_{4}, \lambda_{5}$ & $10^{-2}$ & $10^{-2}$ \\
$\lambda_{6}^{i 2}$ & 0 & 0 \\
\hline
\end{tabular}

Table 3. Mass parameters and couplings used in figure 6 .

which is thus given by

$$
R=D_{M}^{\frac{1}{2}} U_{M} \lambda_{6}^{\top} U_{\nu} D_{\nu}^{-\frac{1}{2}}
$$

Inverting this equation then leads to the Casas-Ibarra parametrisation

$$
\lambda_{6}=U_{\nu}^{*} D_{\nu}^{\frac{1}{2}} R^{\top} D_{M}^{-\frac{1}{2}} U_{M}^{*}
$$

The couplings $\lambda_{6}$ of the dark scalar and fermion sectors are therefore partially determined by the other couplings and masses, encoded in $U_{M}$ and $D_{M}$, and by the measured neutrino mass differences (here: masses, since we assume $m_{\nu_{1}}=0$ and normal hierarchy) $D_{\nu}$ and mixing angles in $U_{\nu}$ [38], but they can still vary with the arbitrary angle $\theta$ in the rotation matrix $R$.

\section{Dark matter direct detection and lepton flavour violation}

In this section, we now connect singlet-doublet fermions with triplet scalars using the couplings $\lambda_{6}$ with the aim to not only explain the observed small neutrino masses as described in the previous section, but also in order to study the effect of the neutrino mass constraints on the nature, allowed parameter space and direct detection prospects of the two DM candidates in this combined model. In addition, we make predictions for the automatically generated lepton flavour violating (LFV) processes and investigate the corresponding current and future experiments with respect to their potential to further restrict the viable parameter space of the model.

For simplicity, we start in figure 6 with the dependence of the relic density on $\lambda_{6}^{i 1}$, i.e. for only one generation of scalars, assuming the couplings to be equal for all three lepton families. The results in figure 6 therefore do not yet yield the correct neutrino masses, but are shown for illustrative purposes only. The mass parameters and other couplings used in this figure are listed in table 3. As described in section 3, two distinct DM candidates give the observed relic density: singlet-doublet fermions $\chi_{1}^{0}$ with masses up to about $1 \mathrm{TeV}$, and triplet scalars with masses around $2 \mathrm{TeV}$. As we can now see, this observation holds also in the case that the masses in the other sector are only larger by $0.2 \mathrm{TeV}$ to $2.5 \mathrm{TeV}$. Only for small mass splittings and very large couplings $\lambda_{6}^{i 1}$, fermion-scalar coannihilation 


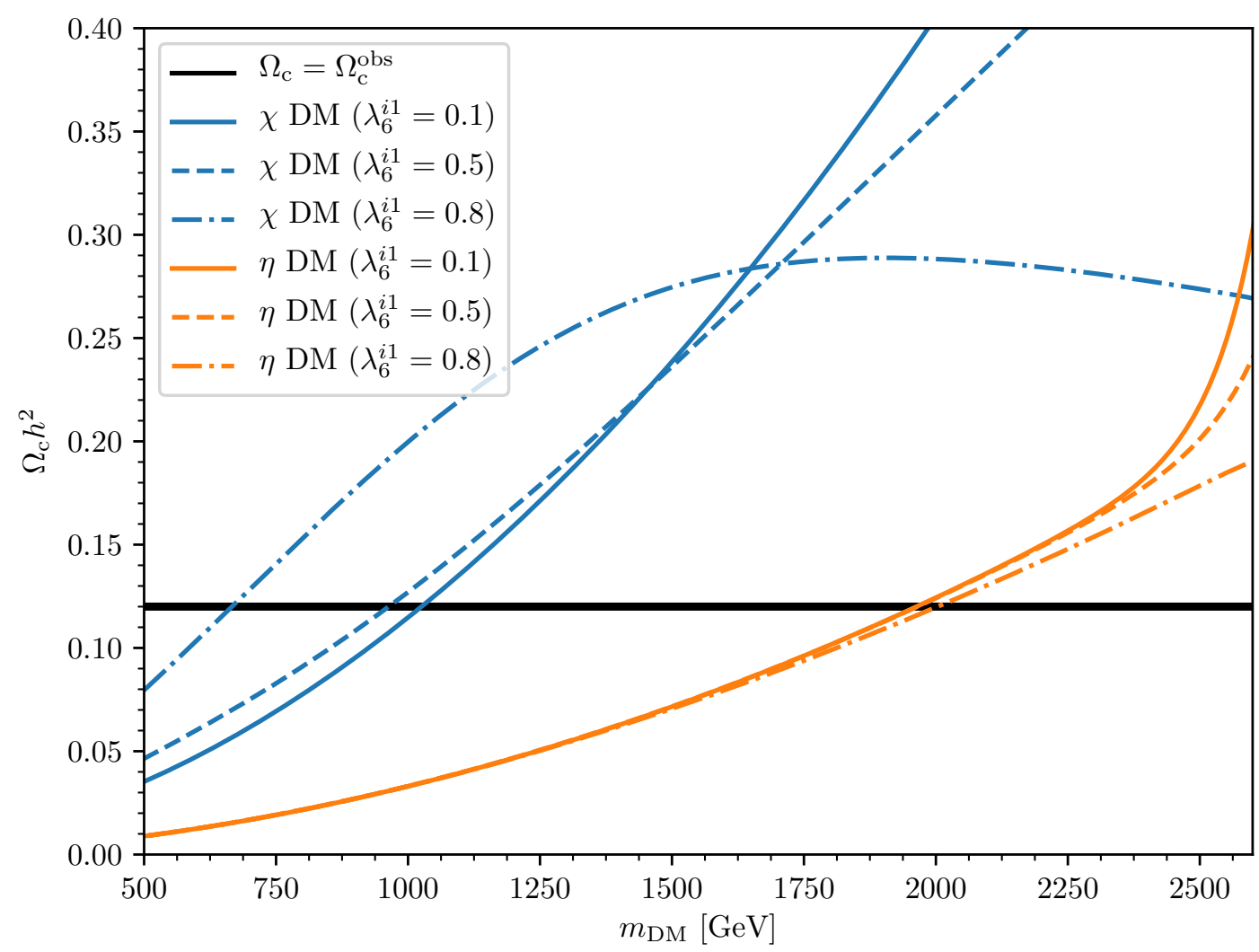

Figure 6. The relic density as a function of the DM mass for singlet-doublet fermion $\left(\chi_{1}^{0}\right)$ and triplet scalar $\left(\eta_{1}^{0}\right)$ DM. The used parameters are given in table 3 .

processes can reduce the relic density, as one can clearly see for triplet scalar DM, which is otherwise insensitive to variations of $\lambda_{6}^{i 1}$. In the heavy scalar mass region, annihilation into weak bosons becomes ineffective, while the one into Higgs bosons remains small due to $\lambda_{1}=0.01$, so that coannihilations can have a significant effect. For fermion DM, it is even more pronounced and starts already at a mass of $1.65 \mathrm{TeV}$, whereas below this value the effect is the opposite and small except for very large $\lambda_{6}^{i 1}=0.8$.

In the following two subsections, we present our main results of a numerical random scan in the parameter space that remains when the observed neutrino mass and mixing constraints as well as the Higgs boson mass $( \pm 2.5 \mathrm{GeV})$ are imposed. In particular, we vary the couplings $\lambda_{1,4,5}$ in the range $\left[10^{-6}, 1\right]$ and the parameters $M_{\phi}, M_{\Psi}$ and $M_{\psi \psi^{\prime}}$ in the range $[10 \mathrm{GeV}, 10000 \mathrm{GeV}] .{ }^{6}$ We also add a random sign for the couplings $\lambda_{1,5}$ while keeping $\lambda_{4}$ always positive [14]. All entries of $\lambda_{1}$ were set to the same value for simplicity (the off-diagonal element only introduces additional mass mixing to the scalar triplets). The coupling $\lambda_{3}$ has no phenomenological implications, and the couplings $\lambda_{6}$ are obtained from the Casas-Ibarra parametrisation as a function of the other parameters. The free

\footnotetext{
${ }^{6}$ Figures $7-12$ show the combined results of two random scans: an exploratory one where the mass parameters $M_{\phi}, M_{\Psi}, M_{\psi \psi^{\prime}}$ were varied in the range $[10 \mathrm{GeV}, 2000 \mathrm{GeV}]$ and one using the full range given above.
} 


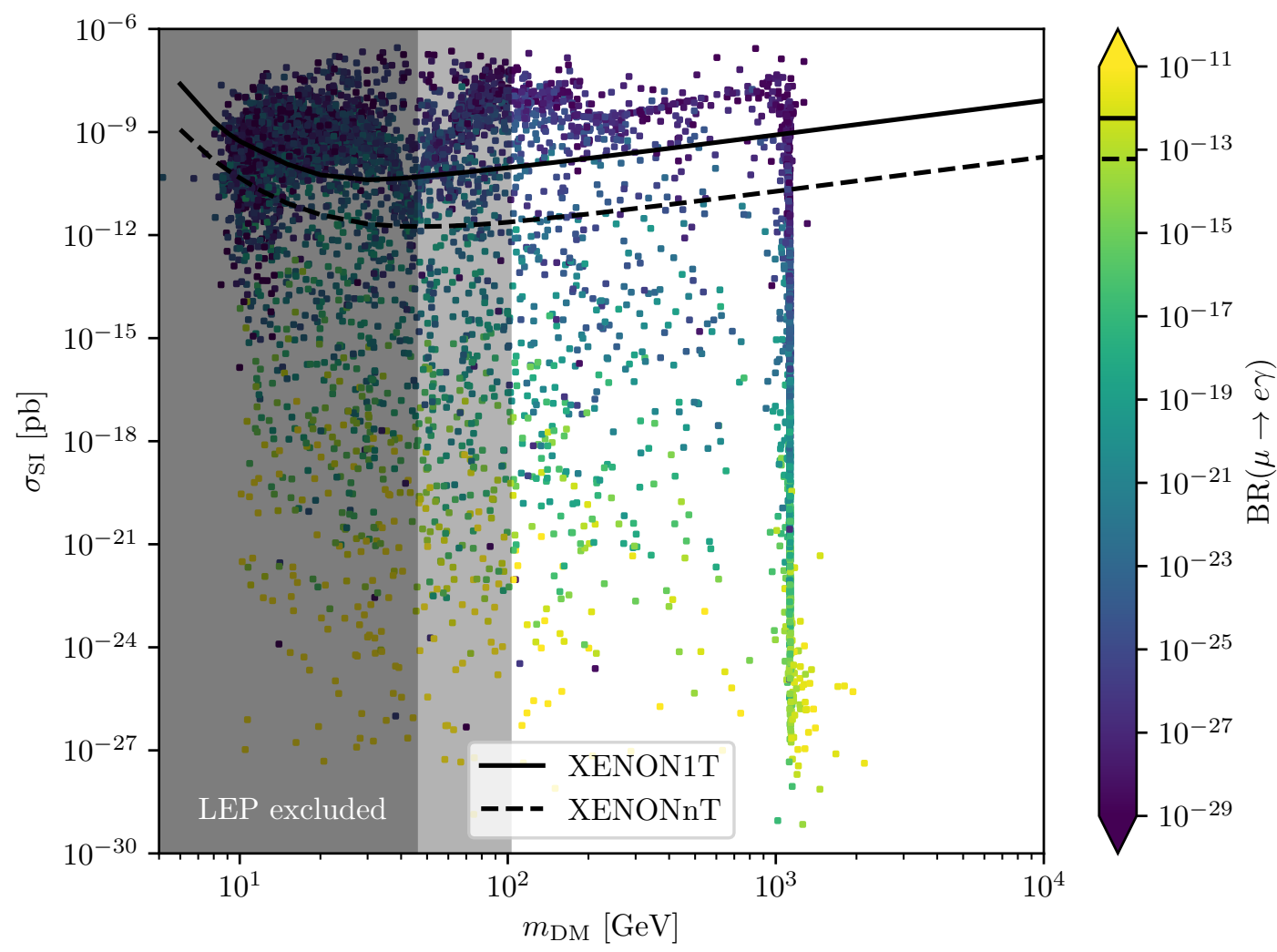

Figure 7. The spin-independent direct detection cross section as a function of the DM mass for singlet-doublet fermion DM. The colours show the branching ratios for the LFV process $\mu \rightarrow e \gamma$. Also shown are the LEP limits on light neutral and charged particles (shaded areas) as well as current (full lines) and future (dashed lines) exclusion limits for the DM relic density from XENON1T [36] and XENONnT [39, 40], and for $\mu \rightarrow e \gamma[41,42]$.

parameter $\theta$ to determine $\lambda_{6}$ is taken randomly in the range $[0,2 \pi]$. Except for the CasasIbarra angle $\theta$, the values of all parameters have been generated from the exponential of randomly-generated numbers with a uniform distribution in the appropriate ranges.

Beyond the results presented in the following, we also checked the impact of electroweak precision data in the form of the $T$ parameter [28] on the model's parameter space. $T$ is defined as exactly zero in the SM and is given there (at tree level) by the expression

$$
\alpha T=\rho-1=\frac{m_{W}^{2}}{m_{Z}^{2} \cos \left(\theta_{\mathrm{w}}\right)} .
$$

For the points which survived all selection criteria (figures 7 and 10), SPheno was used to calculate $T$ to one-loop level. The result is that the vast majority of points yields $T<10^{-20}$, with a maximum value of $T=1.2 \times 10^{-3}$. Compared to the current experimental value of $T=0.07 \pm 0.12$ [28], our results are orders of magnitude below the current bound and therefore $T$ does not currently restrict the parameter space.

\subsection{Singlet-doublet fermion dark matter}

About one third of all models with the observed neutrino masses and mixings feature singlet-doublet fermion DM, but only a fraction of order $0.02 \%$ yield the correct DM relic 
density $\Omega_{\mathrm{c}}^{\text {obs }} h^{2}=0.120 \pm 0.001$ and Higgs mass. These models are shown in figure 7 as a function of the DM mass, together with their spin-independent direct detection cross section and the branching ratio for the usually most sensitive LFV process $\mu \rightarrow e \gamma$. The models accumulating at a DM mass of about $1 \mathrm{TeV}$ feature mostly doublet fermions, whereas lighter fermionic DM is generally a superposition of singlet and doublet (cf. section 3). A large doublet component below $m_{z} / 2$ (dark shaded area) is excluded by the fact that the LEP measurement of the invisible $Z$ boson decay width is consistent with three generations of active neutrinos [28]. We assume this conservative exclusion limit independently of the size of the singlet-doublet mixing. Furthermore, the accompanying, only slightly heavier charged fermions are excluded below $102 \mathrm{GeV}$ by largely model-independent searches with the OPAL detector at LEP (light shaded area) [43]. The LHC limits for heavy long-lived charged particles from ATLAS and CMS reach currently up to $440 \mathrm{GeV}$ and $490 \mathrm{GeV}$, respectively, but are more model-dependent $[44,45]$. LHC signatures and constraints, also from indirect DM detection, up to a few hundred $\mathrm{GeV}$ on singlet-doublet DM without triplet fermions have been discussed in [15]. The spin-independent direct detection cross section is compared to the current XENON1T exclusion limit (full line) [36] and the expectation for 20 ton-years with the XENONnT experiment (dashed line) [39], which was extrapolated linearly above $1 \mathrm{TeV}$ [40]. XENON1T excludes most of the models with small scalar-fermion couplings $\lambda_{6}$ and therefore also little LFV. These models are therefore similar to those in the pure singlet-doublet fermion DM model. The combination with the scalar sector opens up a considerable parameter space of leptophilic DM, i.e. with nuclear recoil cross sections way below even the expected XENONnT sensitivity. Interestingly, one observes a strong complementarity with LFV experiments, which already probe the models with the smallest spin-independent direct detection cross section [41].

Figure 8 shows the models that remain after imposing the XENON1T and LEP limits of $m_{z} / 2$ for neutral scalars [28] and $98 \mathrm{GeV}$ for charged scalars [43] in the mass plane of the lightest neutral fermion (the DM particle $\chi_{1}^{0}$ ) and scalar $\left(\eta_{1}^{0}\right)$. For most models, we observe a near degeneracy of the masses up to $1.1 \mathrm{TeV}$, followed by a line of models with this constant fermion mass and scalar masses up to the decoupling region. These models will not be probed soon by the process $\mu \rightarrow e \gamma$ [42]. Interestingly, there are a few models beyond a fermion mass of $1.1 \mathrm{TeV}$, and these are in fact already excluded by LFV.

In figure 9 we study which of the LFV processes $\mu \rightarrow e \gamma, \mu \rightarrow 3 e$ and $\tau \rightarrow e \gamma$ has the best prospects of further constraining the remaining models. We observe that the three LFV processes are strongly correlated, but that the process $\mu \rightarrow e \gamma$ [41, 42], which is traditionally the most sensitive process, is slightly outrivaled by its competitor $\mu \rightarrow 3 e[46,47]$. In contrast, the process $\tau \rightarrow e \gamma$ has currently no sensitivity to our model $[48,49]$.

\subsection{Triplet scalar dark matter}

About two thirds of all models with the observed neutrino masses and mixings feature triplet scalar DM, but only a fraction of order $0.02 \%$ yield the correct DM relic density $\Omega_{\mathrm{c}}^{\mathrm{obs}} h^{2}=0.120 \pm 0.001$ and Higgs mass. These models are shown in figure 10 as a function of the DM mass, together with their spin-independent direct detection cross section and 


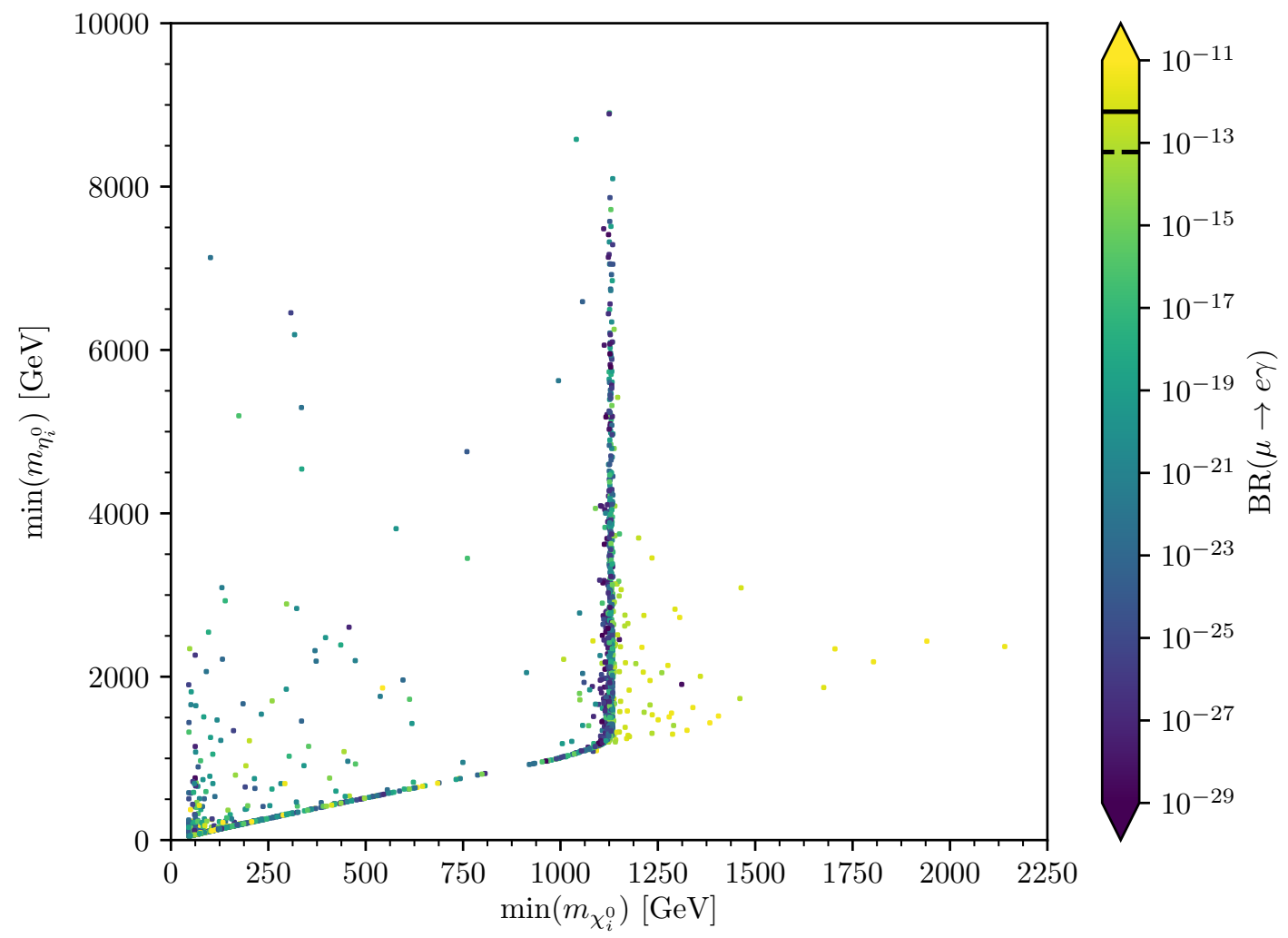

Figure 8. The lightest neutral scalar vs. the lightest neutral fermion mass for viable models with singlet-doublet fermion DM. Also shown is the branching ratio for the LFV process $\mu \rightarrow e \gamma$ with current (full line) and future (dashed line) exclusion limits [41, 42].

the branching ratio for the LFV process $\mu \rightarrow e \gamma$. Similarly to the fermionic DM case, LEP constraints already rule out light DM candidates. The precise measurements of the $Z$ width exclude charged scalars below $m_{z} / 2$ (dark shaded area) and, by virtue of the small mass splitting between charged and neutral components within the same triplet, we can assume this exclusion limit valid also for the neutral scalars, although they do not couple directly to the SM neutral boson because of their vanishing hypercharge. Likewise, the exclusion limit from the aforementioned searches for charged scalars with the OPAL detector is adopted for the neutral components as well, ruling out light scalar DM below $98 \mathrm{GeV}$ [43] (light shaded area). As for a pure triplet scalar model, we observe an accumulation of points around a mass of $2 \mathrm{TeV}$. Many of these models have only very small couplings $\lambda_{6}$ to the fermion sector and thus very little LFV. As $\lambda_{1}$ increases, so must the DM mass beyond $2 \mathrm{TeV}$ to compensate for the stronger Higgs annihilation. However, most of these models will soon be probed by XENONnT, and those that will not can soon be excluded by the process $\mu \rightarrow e \gamma$. While the mass region from $1 \mathrm{TeV}$ to $2 \mathrm{TeV}$ with leptophilic fermion DM, that was opened up by coupling the fermion and scalar sectors, was already excluded by LFV limits (see above), the corresponding models with scalar DM are still allowed, but will soon be probed by the process $\mu \rightarrow e \gamma$. Note that there exists in principle also a region of very light triplet scalar DM of about $6 \mathrm{GeV}$ mass, which is however excluded by the 


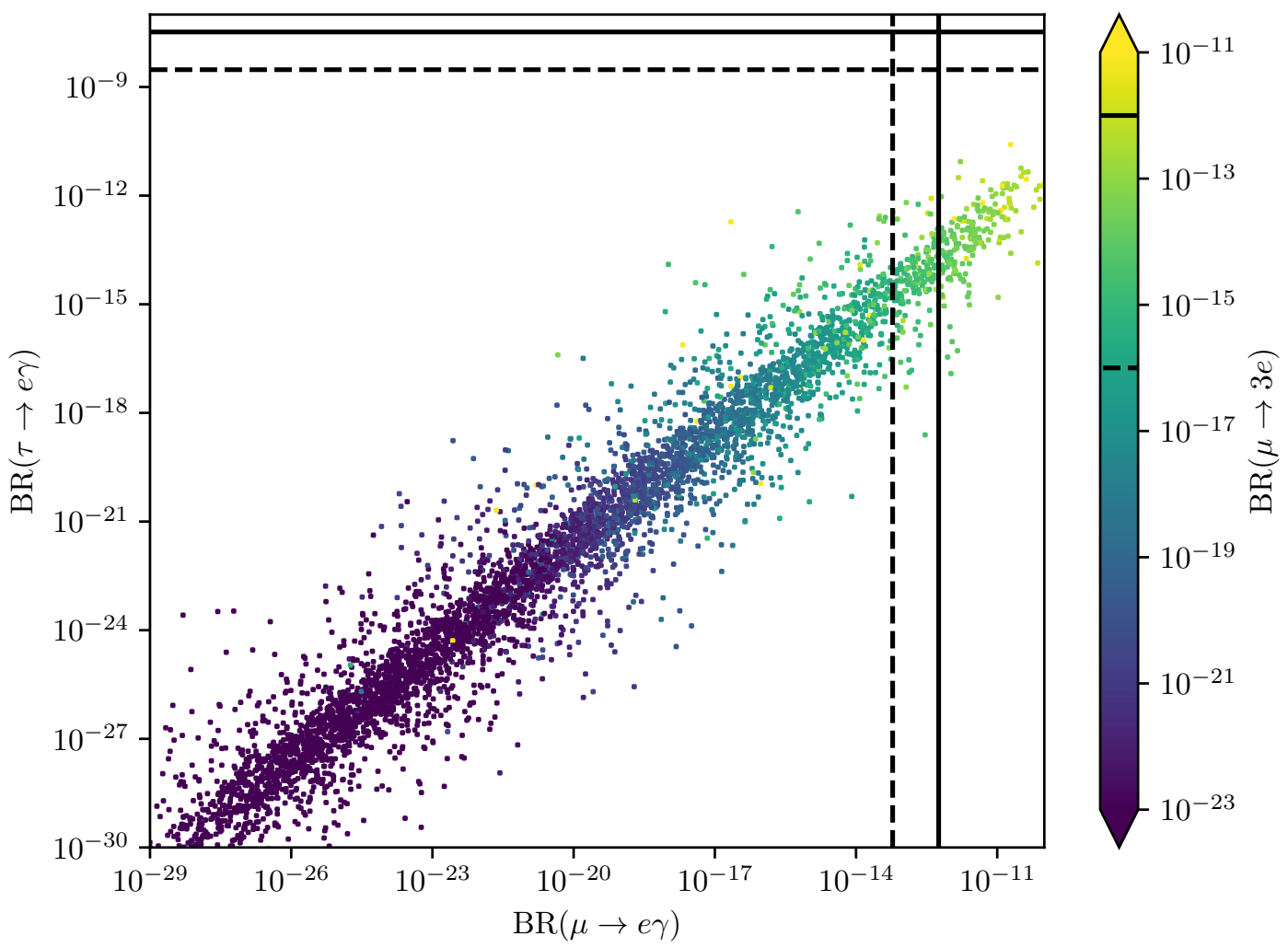

Figure 9. Correlations of the branching ratios for the LFV processes $\mu \rightarrow e \gamma, \mu \rightarrow 3 e$ and $\tau \rightarrow e \gamma$ for viable models with singlet-doublet fermion DM. Also shown are current (full lines) and future (dashed lines) exclusion limits [41, 42, 46-49].

LEP limits on light non-sterile neutral (dark shaded area) and charged (light shaded area) particles.

Figure 11 shows the models that remain after imposing the XENON1T and LEP limits in the mass plane of the lightest neutral scalar (the DM particle $\eta_{1}^{0}$ ) and fermion $\left(\chi_{1}^{0}\right)$. As for fermion DM, we observe a near degeneracy of the masses from $1 \mathrm{TeV}$ to $2 \mathrm{TeV}$, i.e. the two competing DM candidates remain close in mass, but change spin around $1 \mathrm{TeV}$. Despite the small mass difference, coannihilation is rare due to the small couplings $\lambda_{6}$. Once the triplet scalar mass has reached the favoured value of $2 \mathrm{TeV}$, the fermion mass can increase up to the decoupling region. As discussed above, models with higher scalar mass are possible, in particular when the Higgs coupling $\lambda_{1}$ is large, but will soon be probed by either XENONnT or LFV.

Finally, we study the correlation of the three LFV processes $\mu \rightarrow e \gamma, \mu \rightarrow 3 e$ and $\tau \rightarrow e \gamma$ in figure 12 for the case of triplet scalar DM. As for singlet-doublet fermion DM, we observe a strong correlation of the three processes, a similar sensitivity of the two muon decay processes with a small advantage for the one to three electrons, and no sensitivity for the $\tau$ decay. Therefore, in contrast to the fermion DM case, the parameter space for scalar DM can only be fully probed with additional experimental effort. 


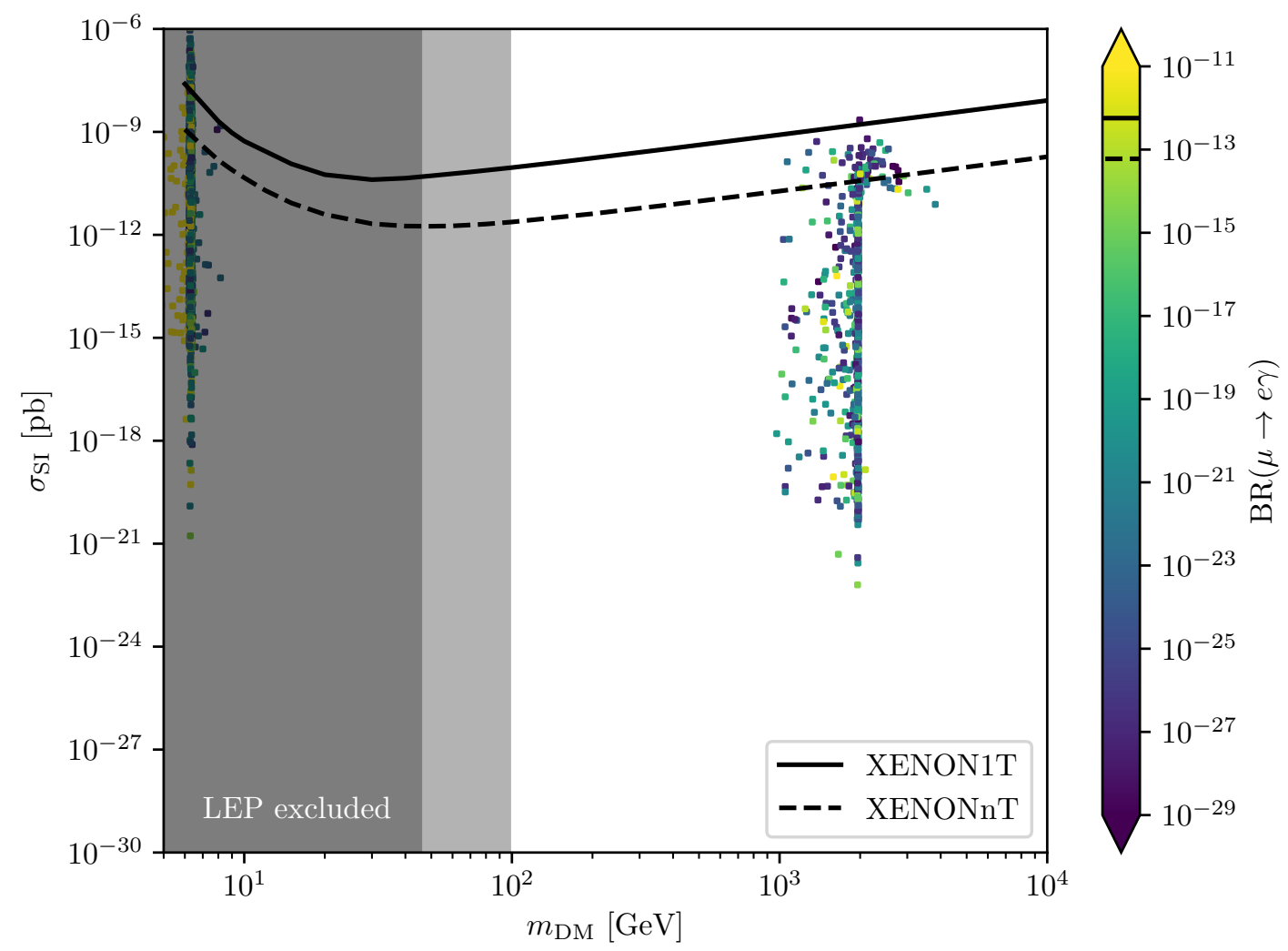

Figure 10. The spin-independent direct detection cross section as a function of the DM mass for triplet scalar DM. The colours show the branching ratios for the LFV process $\mu \rightarrow e \gamma$. Also shown are the LEP limits on light neutral and charged particles (shaded areas) as well as current (full lines) and future (dashed lines) exclusion limits for the DM relic density from XENON1T [36] and XENONnT [39, 40], and for $\mu \rightarrow e \gamma[41,42]$.

\section{Conclusion}

In this paper, we have combined the singlet-doublet fermion model with the triplet scalar model in order to explain not only the observed DM relic density, but also the neutrino masses and mixings, which were generated radiatively. This model allows in addition for the correct Higgs boson mass, couplings of natural size, masses in the $\mathrm{TeV}$ range and gauge coupling unification at a scale of $\mathcal{O}\left(10^{13} \mathrm{GeV}\right)$.

We first updated and clarified some discrepancies in the literature for the two separate DM models, showing that the new XENON1T limits roughly double the excluded parameter space for the singlet-doublet fermion model and confirming that the triplet scalar DM mass is around $2 \mathrm{TeV}$ for small Higgs couplings, but reaches into the multi-TeV region for large Higgs couplings.

With two generations of scalars, we generated two non-zero neutrino masses and implemented the experimental mass and mixing constraints using the Casas-Ibarra parametrisation. Analytically, we found that the product of Yukawa and new scalar-fermion couplings had to be of $\mathcal{O}\left(10^{-5}\right)$ for a DM mass of $1 \mathrm{TeV}$. 


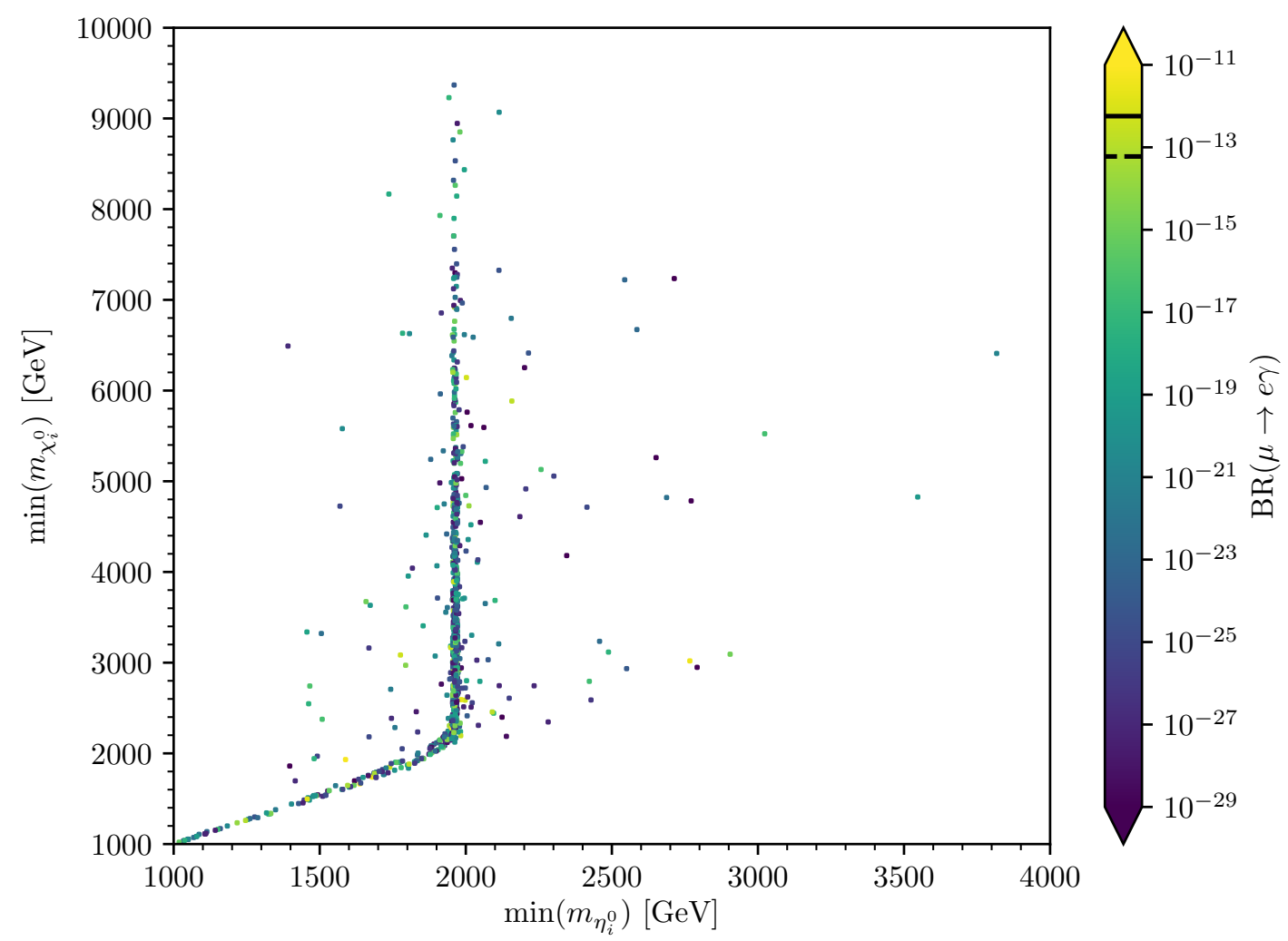

Figure 11. The lightest neutral fermion vs. the lightest neutral scalar mass for viable models with triplet scalar DM. Also shown is the branching ratio for the LFV process $\mu \rightarrow e \gamma$ with current (full line) and future (dashed line) exclusion limits [41, 42]

We found that DM in our model is fermionic up to the TeV scale and scalar beyond with small mass splittings not only among the partners of the weak isospin multiplets, but also the lightest fermions and scalars. The scalar-fermion couplings opened the parameter space, so that leptophilic singlet-doublet fermion DM below $1 \mathrm{TeV}$, but above the LEP limits of $m_{Z} / 2$ for active neutral fermions from the invisible $Z$ boson decay width and $102 \mathrm{GeV}$ from largely model-independent searches for charged fermions, became again viable below the XENON1T exclusion limit, as did triplet scalar DM between $1 \mathrm{TeV}$ and $2 \mathrm{TeV}$. In both regions, we observed an interesting complementarity between the expectations for XENONnT and for LFV experiments. For the latter, the process $\mu \rightarrow 3 e$ has the largest sensitivity, followed by $\mu \rightarrow e \gamma$, whereas or model is insensitive to $\tau \rightarrow e \gamma$. For triplet scalar DM, we found a viable region at masses of a few $\mathrm{GeV}$, which is, however, excluded by LEP limits below $m_{z} / 2$ for neutral and $98 \mathrm{GeV}$ for charged scalars. While current LHC limits reach to considerably higher masses of $440 \mathrm{GeV}$ to $490 \mathrm{GeV}$, they are generally more model-dependent. Triplet scalar DM models with masses above $2 \mathrm{TeV}$ require a large Higgs coupling leading to a large spin-independent cross section that will soon be probed by XENONnT. 


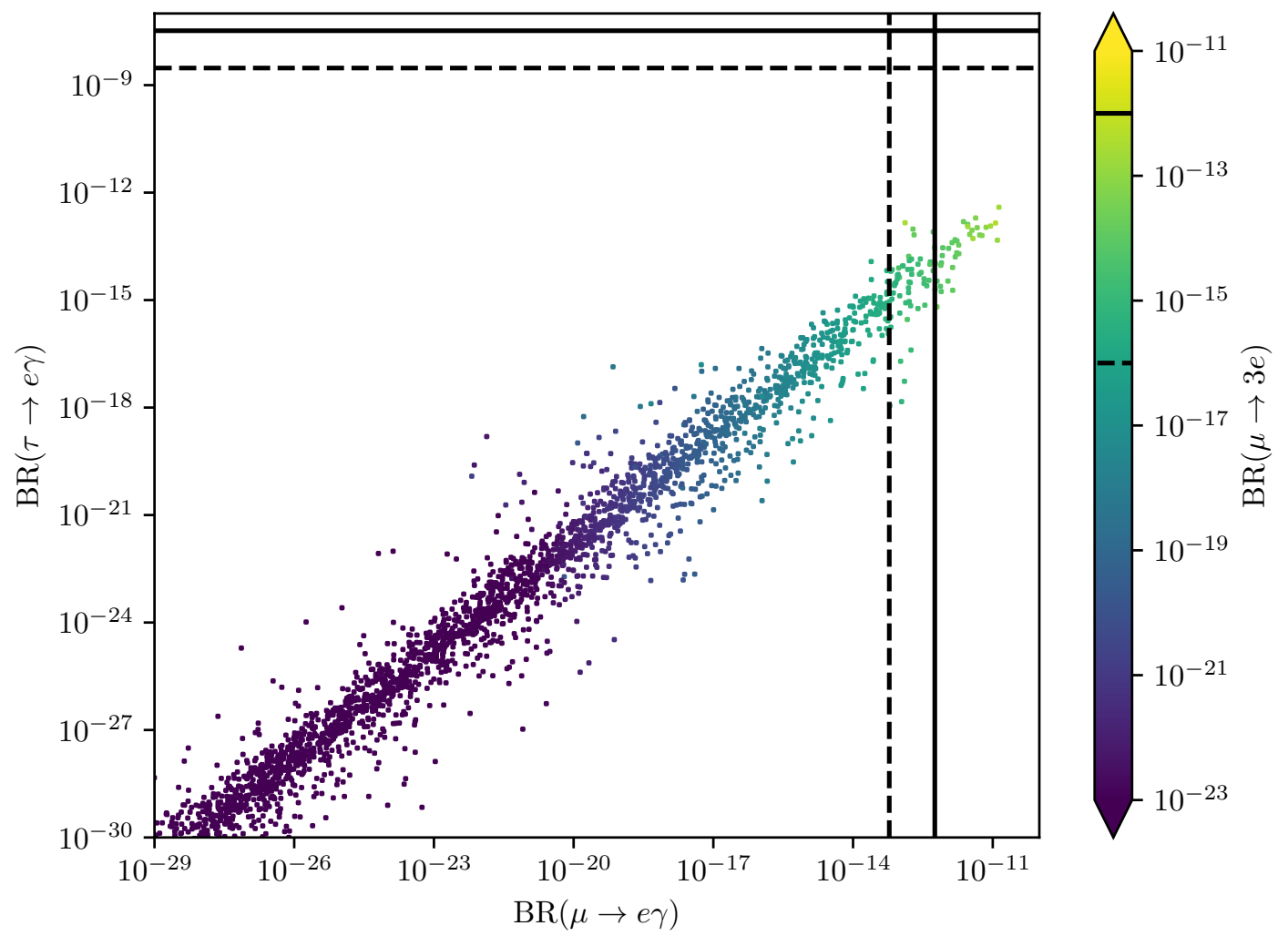

Figure 12. Correlations of the branching ratios for the LFV processes $\mu \rightarrow e \gamma, \mu \rightarrow 3 e$ and $\tau \rightarrow e \gamma$ for viable models with triplet scalar DM. Also shown are current (full lines) and future (dashed lines) exclusion limits [41, 42, 46-49].

\section{Acknowledgments}

This work has been supported by the BMBF under contract 05H18PMCC1 and the DFG through the Research Training Group 2149 "Strong and weak interactions - from hadrons to dark matter". We thank U. Oberlack for communication on the XENONnT expectations and C. Yaguna for helpful comments on the manuscript.

Open Access. This article is distributed under the terms of the Creative Commons Attribution License (CC-BY 4.0), which permits any use, distribution and reproduction in any medium, provided the original author(s) and source are credited.

\section{References}

[1] M. Klasen, M. Pohl and G. Sigl, Indirect and direct search for dark matter, Prog. Part. Nucl. Phys. 85 (2015) 1 [arXiv: 1507.03800] [INSPIRE].

[2] Planck collaboration, Planck 2018 results. VI. Cosmological parameters, arXiv:1807.06209 [INSPIRE].

[3] C.P. Burgess, M. Pospelov and T. ter Veldhuis, The Minimal model of nonbaryonic dark matter: a singlet scalar, Nucl. Phys. B 619 (2001) 709 [hep-ph/0011335] [INSPIRE]. 
[4] L. Lopez Honorez, E. Nezri, J.F. Oliver and M.H.G. Tytgat, The inert doublet model: an archetype for dark matter, JCAP 02 (2007) 028 [hep-ph/0612275] [INSPIRE].

[5] M. Klasen, C.E. Yaguna and J.D. Ruiz-Alvarez, Electroweak corrections to the direct detection cross section of inert Higgs dark matter, Phys. Rev. D 87 (2013) 075025 [arXiv: 1302.1657] [INSPIRE].

[6] T. Araki, C.Q. Geng and K.I. Nagao, Dark matter in inert triplet models, Phys. Rev. D 83 (2011) 075014 [arXiv:1102.4906] [INSPIRE].

[7] O. Fischer and J.J. van der Bij, Multi-singlet and singlet-triplet scalar dark matter, Mod. Phys. Lett. A 26 (2011) 2039 [inSPIRE].

[8] F.-X. Josse-Michaux and E. Molinaro, Triplet scalar dark matter and leptogenesis in an inverse seesaw model of neutrino mass generation, Phys. Rev. D 87 (2013) 036007 [arXiv: 1210.7202] [INSPIRE].

[9] S. Yaser Ayazi and S.M. Firouzabadi, Footprint of triplet scalar dark matter in direct, indirect search and invisible Higgs decay, Cogent Phys. 2 (2015) 1047559 [arXiv:1501.06176].

[10] N. Khan, Exploring the hyperchargeless Higgs triplet model up to the Planck scale, Eur. Phys. J. C 78 (2018) 341 [arXiv:1610.03178] [INSPIRE].

[11] S. Esch, M. Klasen and C.E. Yaguna, Detection prospects of singlet fermionic dark matter, Phys. Rev. D 88 (2013) 075017 [arXiv:1308.0951] [INSPIRE].

[12] M. Klasen and C.E. Yaguna, Warm and cold fermionic dark matter via freeze-in, JCAP 11 (2013) 039 [arXiv: 1309.2777] [INSPIRE].

[13] T. Cohen, J. Kearney, A. Pierce and D. Tucker-Smith, Singlet-doublet dark matter, Phys. Rev. D 85 (2012) 075003 [arXiv: 1109.2604] [INSPIRE].

[14] C. Cheung and D. Sanford, Simplified models of mixed dark matter, JCAP 02 (2014) 011 [arXiv: 1311.5896] [INSPIRE].

[15] L. Calibbi, A. Mariotti and P. Tziveloglou, Singlet-doublet model: dark matter searches and LHC constraints, JHEP 10 (2015) 116 [arXiv:1505.03867] [INSPIRE].

[16] A. Dedes and D. Karamitros, Doublet-triplet fermionic dark matter, Phys. Rev. D 89 (2014) 115002 [arXiv: 1403.7744] [INSPIRE].

[17] T. Hambye, F.S. Ling, L. Lopez Honorez and J. Rocher, Scalar multiplet dark matter, JHEP 07 (2009) 090 [Erratum ibid. 05 (2010) 066] [arXiv:0903.4010] [INSPIRE].

[18] M. Cirelli, N. Fornengo and A. Strumia, Minimal dark matter, Nucl. Phys. B 753 (2006) 178 [hep-ph/0512090] [INSPIRE].

[19] D. Restrepo, O. Zapata and C.E. Yaguna, Models with radiative neutrino masses and viable dark matter candidates, JHEP 11 (2013) 011 [arXiv:1308.3655] [INSPIRE].

[20] F. Bonnet, M. Hirsch, T. Ota and W. Winter, Systematic study of the $d=5$ Weinberg operator at one-loop order, JHEP 07 (2012) 153 [arXiv: 1204.5862] [INSPIRE].

[21] E. Ma, Verifiable radiative seesaw mechanism of neutrino mass and dark matter, Phys. Rev. D 73 (2006) 077301 [hep-ph/0601225] [INSPIRE].

[22] M. Klasen et al., Scalar dark matter and fermion coannihilations in the radiative seesaw model, JCAP 04 (2013) 044 [arXiv: 1302.5298] [INSPIRE]. 
[23] S.S.C. Law and K.L. McDonald, A class of inert N-tuplet models with radiative neutrino mass and dark matter, JHEP 09 (2013) 092 [arXiv:1305.6467] [INSPIRE].

[24] V. Brdar, I. Picek and B. Radovcic, Radiative neutrino mass with scotogenic scalar triplet, Phys. Lett. B 728 (2014) 198 [arXiv:1310.3183] [InSPIRE].

[25] Y. Farzan, S. Pascoli and M.A. Schmidt, AMEND: a model explaining neutrino masses and dark matter testable at the LHC and MEG, JHEP 10 (2010) 111 [arXiv:1005.5323] [INSPIRE].

[26] H. Okada and Y. Orikasa, Radiative neutrino model with an inert triplet scalar, Phys. Rev. D 94 (2016) 055002 [arXiv: 1512.06687] [INSPIRE].

[27] S. Esch, M. Klasen, D.R. Lamprea and C.E. Yaguna, Lepton flavor violation and scalar dark matter in a radiative model of neutrino masses, Eur. Phys. J. C 78 (2018) 88 [arXiv: 1602.05137] [INSPIRE].

[28] Particle Data Group collaboration, Review of particle physics, Phys. Rev. D 98 (2018) 030001 [INSPIRE].

[29] S. Esch, M. Klasen and C.E. Yaguna, A singlet doublet dark matter model with radiative neutrino masses, JHEP 10 (2018) 055 [arXiv: 1804.03384] [INSPIRE].

[30] C. Hagedorn, T. Ohlsson, S. Riad and M.A. Schmidt, Unification of gauge couplings in radiative neutrino mass models, JHEP 09 (2016) 111 [arXiv:1605.03986] [INSPIRE].

[31] F. Staub, SARAH 4: a tool for (not only SUSY) model builders, Comput. Phys. Commun. 185 (2014) 1773 [arXiv: 1309.7223] [INSPIRE].

[32] J.A. Casas and A. Ibarra, Oscillating neutrinos and $\mu \rightarrow e \gamma$, Nucl. Phys. B 618 (2001) 171 [hep-ph/0103065] [INSPIRE].

[33] W. Porod and F. Staub, SPheno 3.1: extensions including flavour, CP-phases and models beyond the MSSM, Comput. Phys. Commun. 183 (2012) 2458 [arXiv:1104.1573] [INSPIRE].

[34] D. Barducci et al., Collider limits on new physics within MicrOMEGAs_4.3, Comput. Phys. Commun. 222 (2018) 327 [arXiv: 1606. 03834] [INSPIRE].

[35] J.F. Gunion and H.E. Haber, The CP conserving two Higgs doublet model: The Approach to the decoupling limit, Phys. Rev. D 67 (2003) 075019 [hep-ph/0207010] [INSPIRE].

[36] XENON collaboration, Dark matter search results from a one ton-year exposure of XENON1T, Phys. Rev. Lett. 121 (2018) 111302 [arXiv:1805.12562] [INSPIRE].

[37] H.K. Dreiner, H.E. Haber and S.P. Martin, Two-component spinor techniques and Feynman rules for quantum field theory and supersymmetry, Phys. Rept. 494 (2010) 1 [arXiv: 0812.1594] [INSPIRE].

[38] M.C. Gonzalez-Garcia, M. Maltoni and T. Schwetz, Updated fit to three neutrino mixing: status of leptonic CP-violation, JHEP 11 (2014) 052 [arXiv: 1409.5439] [INSPIRE].

[39] XENON collaboration, Physics reach of the XENON1T dark matter experiment, JCAP 04 (2016) 027 [arXiv : 1512.07501] [INSPIRE].

[40] U. Oberlack, private communication.

[41] MEG collaboration, New constraint on the existence of the $\mu^{+} \rightarrow e^{+} \gamma$ decay, Phys. Rev. Lett. 110 (2013) 201801 [arXiv: 1303.0754] [INSPIRE].

[42] A.M. Baldini et al., MEG upgrade proposal, arXiv:1301.7225 [INSPIRE]. 
[43] OPAL collaboration, Search for stable and longlived massive charged particles in $e^{+} e^{-}$ collisions at $\sqrt{s}=130$ GeV to 209 GeV, Phys. Lett. B 572 (2003) 8 [hep-ex/0305031] [INSPIRE].

[44] ATLAS collaboration, Searches for heavy long-lived charged particles with the ATLAS detector in proton-proton collisions at $\sqrt{s}=8 \mathrm{TeV}$, JHEP 01 (2015) 068 [arXiv:1411.6795] [INSPIRE].

[45] CMS collaboration, Search for long-lived charged particles in proton-proton collisions at $\sqrt{s}=13$ TeV, Phys. Rev. D 94 (2016) 112004 [arXiv:1609.08382] [INSPIRE].

[46] SINDRUM collaboration, Search for the decay $\mu^{+} \rightarrow e^{+} e^{+} e^{-}$, Nucl. Phys. B 299 (1988) 1 [INSPIRE].

[47] A. Blondel et al., Research proposal for an experiment to search for the decay $\mu \rightarrow$ eee, arXiv:1301.6113 [INSPIRE].

[48] BABAR collaboration, Searches for lepton flavor violation in the decays $\tau^{ \pm} \rightarrow e^{ \pm} \gamma$ and $\tau \pm \rightarrow \mu^{ \pm} \gamma$, Phys. Rev. Lett. 104 (2010) 021802 [arXiv:0908.2381] [INSPIRE].

[49] T. Aushev et al., Physics at super B factory, arXiv:1002.5012 [InSPIRE]. 